\title{
One-Year Results of Treatment of Diabetic Macular Edema with Aflibercept Using the Treat-and-Extend Dosing Regimen: the VIBIM Study
}

\author{
Kang Yeun Pak ${ }^{\mathrm{a}}$ Jae Pil Shin ${ }^{\mathrm{b}}$ Hyun Woong Kim ${ }^{\mathrm{a}}$ Min Sagong ${ }^{\mathrm{c}}$ \\ Yu Cheol Kim ${ }^{d}$ Sang Joon Lee ${ }^{e}$ In Young Chung ${ }^{f}$ Sung Who Park ${ }^{g, h}$ \\ Ji Eun Lee ${ }^{g, h}$
}

${ }^{a}$ Department of Ophthalmology, Haeundae Paik Hospital, Inje University College of Medicine, Busan, South Korea; ${ }^{b}$ Department of Ophthalmology, Kyungpook National University School of Medicine, Deagu, South Korea; ${ }^{\circ}$ Department of Ophthalmology, Yeungnam University College of Medicine, Daegu, South Korea; ${ }^{\mathrm{D}}$ Department of Ophthalmology, Dongsan Medical Center, Keimyung University School of Medicine, Daegu, South Korea; ${ }^{e}$ Department of Ophthalmology, Kosin University College of Medicine, Busan, South Korea; ${ }^{f}$ Department of Ophthalmology, Gyeongsang National University School of Medicine, Jinju, South Korea; ${ }^{9}$ Department of Ophthalmology, Pusan National University, College of Medicine, Yangsan, South Korea; ${ }^{\mathrm{h}}$ Biomedical Research Institute, Pusan National University Hospital, Busan, South Korea

\section{Keywords}

Aflibercept · Diabetic macular edema - Treat-and-extend regimen

\begin{abstract}
Introduction: The purpose of this study was to report the one-year results of treatment of diabetic macular edema (DME) with aflibercept using a treat-and-extend regimen (TER). Methods: This was a prospective, multicenter, singlearm study planned for 2 years. The eyes received 5 consecutive intravitreal injections of $2 \mathrm{mg}$ of aflibercept every 4 weeks, and the interval between injections was then adjusted by 2 weeks based on changes in the central subfield macular thickness (CSMT). If the CSMT was worse, stable, or better, the interval was shortened, extended, or maintained, respectively. The primary outcome measure was the change in best-corrected visual acuity (BCVA) from baseline to 104 weeks, and the secondary outcome was the change in BCVA from baseline to 52 weeks. Results: Of the 48 patients enrolled, 46 completed a 1-year visit. BCVA improved significantly by 9.1 letters (95\% confidence interval: $5.3-13.0$ letters) from 56.2 letters at baseline $(p<0.001)$, and CSMT de-
\end{abstract}

$\begin{aligned} & \text { karger@karger.com } \\ & \text { www.karger.com/oph }\end{aligned}$
Karger $\%$

creased by $-171.7 \mu \mathrm{m}$ from 489.4 to $317.7 \mu \mathrm{m}(p<0.001)$. The proportion of eyes having $20 / 40$ or better vision increased from 17.4 to $41.3 \%$, and the proportion of eyes that gained $\geq 15$ letters was $28.3 \%$. The mean number of injections was 8.5 times for 52 weeks. Worsening of macular edema did not occur in $76.1 \%$ of eyes during the extension period, and the interval between injections was extended to 12 weeks in $73.9 \%$ of eyes at 52 weeks. Conclusions: The TER showed 1-year efficacy comparable to that of the fixed dosing regimen of pivotal trials, and its flexible dosing would prevent overtreatment.

๑ 2020 S. Karger AG, Basel

\section{Introduction}

Diabetic macular edema (DME) is the leading cause of visual loss related to diabetic retinopathy [1]. Originally, focal laser treatment [2] and intravitreal or periocular corticosteroids $[3,4]$ were widely accepted as the standard treatments for DME. This was followed by the era of anti-vascular endothelial growth factor (anti-VEGF), which began with the introduction of ranibizumab; its ef- 
ficacy was shown in the RISE and RIDE study [5]. Although the risk of blindness has been reduced, a fixed dosing regimen of monthly intravitreal injections is quite strenuous for most patients and doctors, and quite burdensome for the healthcare system as well [6].

Aflibercept is a chimeric protein containing key domains from human VEGF receptors and has binding affinity to VEGF-A around 100-fold greater than other anti-VEGF agents [7]. The VISTA and VIVID studies demonstrated that the anatomic and functional outcomes achieved with aflibercept injections of $2 \mathrm{mg}$ every 8 weeks (2q8) for DME were comparable to injections of $2 \mathrm{mg} \mathrm{ev}$ ery 4 weeks (2q4) and superior to the outcomes of focal laser treatment $[8,9]$. Nevertheless, a fixed dosing regimen may still run the risk of potential overtreatment and is a burden to maintain.

With specific regard to reducing the treatment cost associated with the fixed dosing regimen, variable dosing regimens were introduced in age-related macular degeneration (ARMD) before DME $[10,11]$. In a pro re nata $(\mathrm{PRN})$ regimen, treatment is decided based on disease activity; this approach can reduce the number of required drug doses. However, a PRN regimen still requires frequent visits and the long-term effects of permitting recurrence are still unclear. By contrast, a treat-and-extend regimen (TER) adjusts the intervals between visits at each visit. It is gaining popularity for the management of ocular diseases requiring repeated anti-VEGF injections [12, 13]. The TER has shown noninferior results compared with fixed dosing in exudative ARMD with fewer injections and visits $[14,15]$. The efficacy of the TER using ranibizumab was also reported for DME in the RETAIN and TREX-DME studies [16, 17]. However, to the best of our knowledge, there has been no report on the treatment of DME with a TER using aflibercept.

This study (the treat-and-extend regimen using intraVItreal afliBercept In diabetic Macular edema [VIBIM] study) was designed to evaluate the efficacy of the TER using aflibercept in the management of DME for a period of 2 years. The results of the 1-year interim analysis are presented here.

\section{Subjects and Methods}

\section{Study Design}

The VIBIM study was designed as a phase 4, prospective, multicenter, interventional, single-arm, 104-week clinical trial (ClinicalTrials.gov ID, NCT02788877). Eight centers in South Korea enrolled 48 eyes with DME.
Inclusion and Exclusion Criteria

Adult patients aged $\geq 19$ years with type 1 or 2 diabetes mellitus who presented with DME involving the fovea, with a central subfield macular thickness (CSMT) $\geq 300 \mu \mathrm{m}$ on optical coherence tomography (OCT), were eligible for enrollment if their best-corrected visual acuity (BCVA) was between 20/40 and 20/320 (Snellen visual acuity [VA]). If both eyes were eligible, only 1 eye per patient was enrolled in the study at the investigator's discretion.

Key exclusion criteria were as follows: (1) a history of vitreoretinal surgery in the study eye, (2) laser photocoagulation in the study eye within 90 days of enrollment, (3) previous use of an intra-/periocular steroid in the study eye within 120 days of enrollment, and (4) previous use of intraocular anti-VEGF in the study eye within 90 days of enrollment.

\section{Treatment and Assessment Schedule}

Patients were treated according to the TER protocol. Eyes received 5 consecutive intravitreal injections of $2 \mathrm{mg}$ aflibercept every 4 weeks during the "loading phase," after which the treatment entered the "TnE phase" (treat-and-extend phase). The interval between injections was adjusted by 2 weeks based on changes of CSMT in the "TnE phase." This adjustment was done using a threshold of $10 \%$ change (decrease or increase) in CSMT compared to that of the previous visit. If the CSMT was worse, stable, or better, the interval was shortened (minimum 4 weeks), extended, or maintained (maximum 12 weeks), respectively (Fig. 1). During the loading phase, a patient was allowed to enter the TnE phase if the CSMT was $<250$ $\mu \mathrm{m}$ and the Snellen VA was 20/20 before the 5 consecutive injections. During the TnE phase, if the CSMT was stable for 2 consecutive visits and the interval between visits had been extended to the maximum of 12 weeks, the treatment was deferred and the next interval was shortened to 8 weeks. If there was no worsening of DME, the patient was observed without treatment at 8 -week intervals. The treatment schedule was managed using online software by submitting the VA and CSMT records through the web program to avoid subjective error caused by the researchers.

At each visit, full ophthalmic examinations were performed including BCVA measurement using the Early Treatment Diabetic Retinopathy Study (ETDRS) chart, measurement of intraocular pressure, slit lamp examination, color fundus photography, and spectral domain OCT. Fluorescein angiography was scheduled to be performed at baseline and at 52 and 104 weeks. Diabetic retinopathy severity scale (DRSS) was evaluated according to the ETDRS scale for retinopathy severity and graded as a low (DRSS score $\leq 43$ ), moderate (DRSS score 47), or high (DRSS score $\geq 53$ ) risk.

Focal laser treatment or intravitreal triamcinolone acetonide injection were permitted as a rescue treatment at the treating physician's discretion, only if CSMT increased by up to $10 \%$ from baseline and VA decreased due to DME by $>10$ ETDRS chart letters at 24 weeks or later.

\section{Outcome Measures}

The primary outcome measure was the change in BCVA in ETDRS letter score at 104 weeks, and the secondary outcomes included changes in ETDRS letter score at 52 weeks and various parameters (CSMT changes, number of injections, mean interval between injections, the proportion of eyes with an injection interval of 12 weeks, the proportion of eyes with a gain of 15 letters, the proportion of eyes having 20/50 or better vision, and DRSS score changes) at 52 and 104 weeks. This report presents the interim results at 52 weeks. 


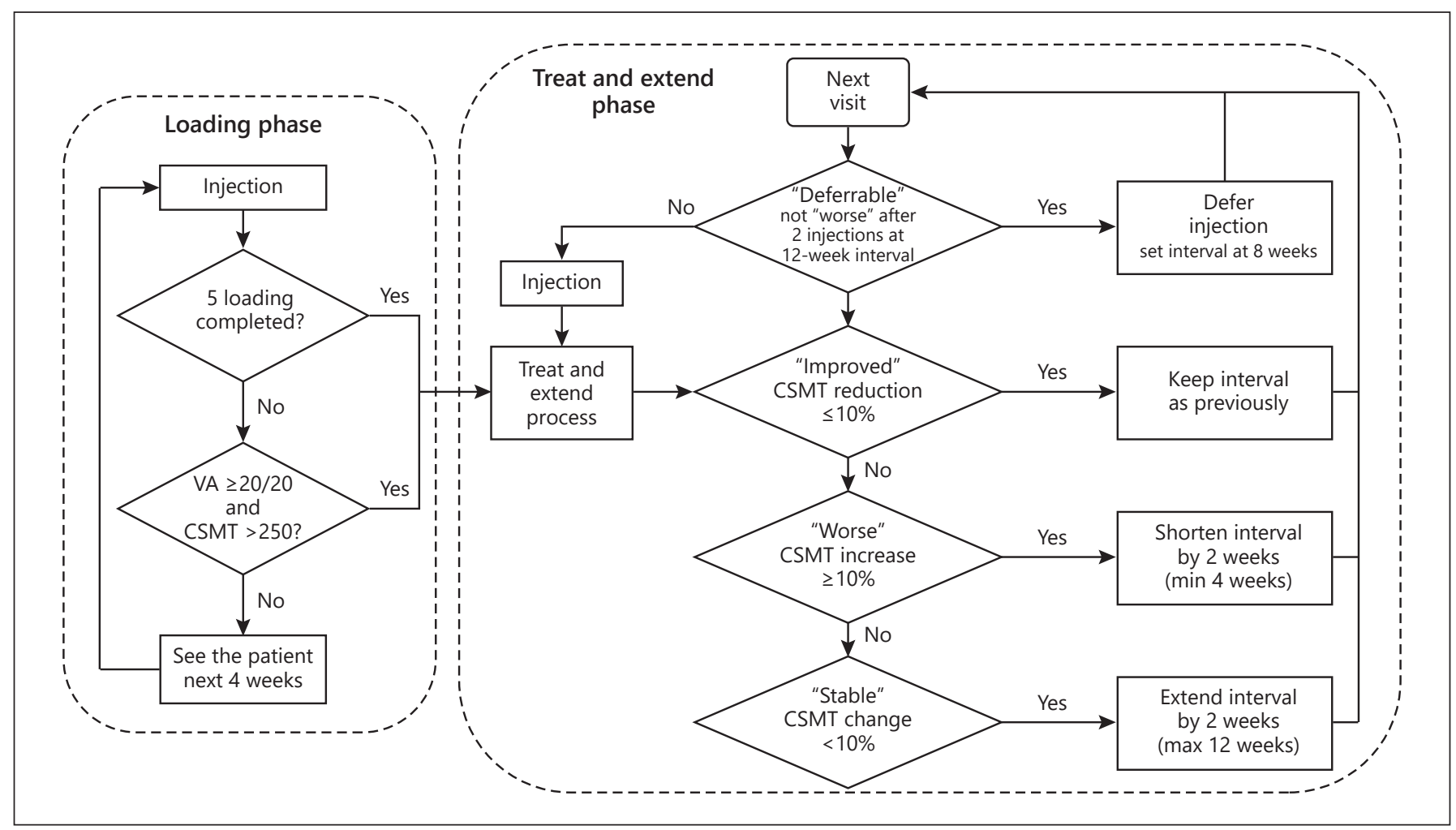

Fig. 1. The flowchart demonstrates the treat-and-extend regimen using aflibercept for the treatment of diabetic macular edema.

\section{Statistical Analysis}

Wilcoxon signed-rank or Mann-Whitney U nonparametric tests were used to compare the measured values. Categorical variables were analyzed using the $\chi^{2}$ test or Fisher's exact test. Statistical significance was defined as $p<0.05$. IBM SPSS Statistics for Windows (v23.0, IBM Corp., Armonk, NY, USA) was used to perform statistical analyses.

\section{Results}

\section{Functional and Anatomical Outcomes}

The study enrolled 48 patients, and 46 patients ( 23 males and 23 females) completed a 52 -week visit. Two patients dropped out by withdrawing their consent. The mean age of included subjects was $59.4 \pm 12.4$ years. Mean duration of diabetes was $16.6 \pm 8.5$ years, and 20 patients (43.5\%) were treatment-naïve. The baseline characteristics are shown in Table 1.

Mean BCVA improved from $56.2 \pm 16.4$ at baseline to $65.3 \pm 15.4$ at 52 weeks $(+9.1 \pm 12.7$ letters, $95 \%$ confidence interval 5.3-13.0 letters, $p<0.001$; Fig. 2a). The significant BCVA improvement was noted from 4 weeks
$(+5.0 \pm 9.7$ letters, $p<0.001)$ throughout the study period to 52 weeks. The proportion of eyes that gained or lost $\geq 15$ letters was 28.3 and $4.3 \%$, respectively (Fig. $3 a$ ). Visual gain of $\geq 10$ letters was noted in $54.3 \%$. The eyes with BCVA $\geq 20 / 40$ increased from $17.4 \%$ at baseline to $45.7 \%$ at 52 weeks ( $p=0.007, \chi^{2}$; Fig. 3b).

Mean CSMT, as measured by OCT, significantly decreased from $489.4 \pm 131.4 \mu \mathrm{m}$ at baseline to $317.7 \pm 83.2$ $\mu \mathrm{m}$ at 52 weeks (mean improvement $-171.7 \pm 139.3 \mu \mathrm{m}$; Fig. 2b).

For 52 weeks, the mean number of injections was 8.5 \pm 0.8 (median 8 , range $8-12$ ). Worsening of macular edema was noted in $23.9 \%$ of eyes, and the interval between injections was extended to 12 weeks in $73.9 \%$ of eyes. The mean interval between injections was 10.7 weeks at 52 weeks.

DRSS score improved from $40.9 \%$ (a low risk), 20.5\% (a moderate risk), and $38.6 \%$ (a high risk) at baseline to $71.4,11.9$, and $16.7 \%$ at 52 weeks, respectively. The proportion of eyes that showed $\geq 2$-step regression in DRSS level was $27.5 \%$. 

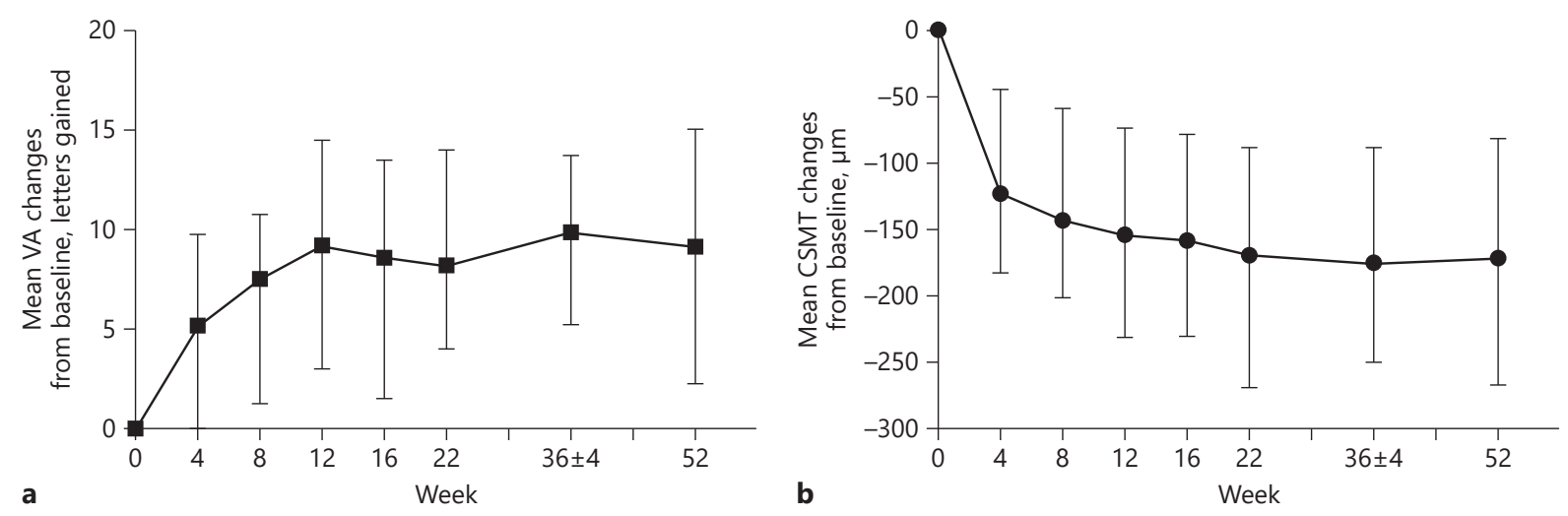

Fig. 2. Visual acuity (VA) (a) and central subfield macular thickness (CSMT) (b) changes for 52 weeks in diabetic macular edema treated with aflibercept using the treat-and-extend regimen. Statistically significant differences were noted from 4 weeks ( $p<0.05$, Wilcoxon signed-rank test).

Table 1. Baseline characteristics of diabetic macular edema patients treated with aflibercept using the treat-andextend regimen

\begin{tabular}{lcccc}
\hline & $\begin{array}{l}\text { Total } \\
(n=46)\end{array}$ & Subgroup & & \\
\cline { 3 - 5 } & & $\begin{array}{l}\text { Recur }(-) \\
(n=35)\end{array}$ & $\begin{array}{l}\text { Recur }(+) \\
(n=11)\end{array}$ & $p$ value \\
\hline Age, years & $59.4 \pm 12.2$ & $60.8 \pm 12.3$ & $55.2 \pm 11.3$ & 0.252 \\
Males:females & $23: 23(50: 50)$ & $17: 18(49: 51)$ & $6: 5(55: 45)$ & 1.0 \\
DM duration, years & $16.6 \pm 8.5$ & $16.5 \pm 8.9$ & $17.0 \pm 7.3$ & 0.718 \\
Treatment-naïve, $\%$ & $20(43.5)$ & $16(45.7)$ & $4(36.4)$ & 0.732 \\
Previous PRP, \% & $23(50.0)$ & $15(42.9)$ & $8(72.7)$ & 0.165 \\
HbA 1 \% $\%$ & $7.8 \pm 1.5$ & $7.6 \pm 1.5$ & $8.4 \pm 1.5$ & 0.168 \\
BUN, mg/dL & $19.7 \pm 10.1$ & $20.1 \pm 9.2$ & $18.3 \pm 11.3$ & 0.313 \\
Creatinine, mg/dL & $1.1 \pm 0.6$ & $1.1 \pm 0.6$ & $0.9 \pm 0.2$ & 0.813 \\
LDL, mg/dL & $90.5 \pm 37.0$ & $93.3 \pm 34.3$ & $80.9 \pm 33.9$ & 0.224 \\
TG, mg/dL & $160.7 \pm 89.0$ & $145 \pm 73.8$ & $213 \pm 119.1$ & 0.086 \\
VA, $n$ of letters gained & $56.2 \pm 16.4$ & $57.8 \pm 14.3$ & $50.9 \pm 21.7$ & 0.284 \\
CSMT, $\mu$ m & $489.4 \pm 130.4$ & $467.1 \pm 117.8$ & $560.4 \pm 148.4$ & 0.080 \\
\hline
\end{tabular}

Values express mean \pm SD or $n(\%)$. DM, diabetes mellitus; PRP, panretinal photocoagulation; BUN, blood urea nitrogen; LDL, low-density lipoprotein; TG, triglyceride; VA, visual acuity; CSMT, central subfield macular thickness.

There was no severe adverse event related to the study drug or procedures. Two cases had a loss of $\geq 15$ letters at 52 weeks, one due to cataract progression and the other related to acute worsening of macular edema that occurred after extending the treatment interval.

\section{Subgroup Analysis according to Recurrence}

Patients were divided into 2 subgroups according to episodes of worsened macular edema in the TnE phase: a
Recur (-) group ( $n=35)$ and Recur $(+)$ group $(n=11)$. Baseline characteristics and treatment outcomes were compared between these 2 groups, and no significant differences were found in baseline characteristics including BCVA, CSMT, and a previous history of panretinal photocoagulation (Table 1). At 52 weeks, BCVA changed by $10.3 \pm 11.4$ letters in the Recur $(-)$ group and $5.4 \pm 16.2$ letters in the Recur (+) group, with no significant differences between the 2 groups ( $p=0.346)$. However, BCVA 

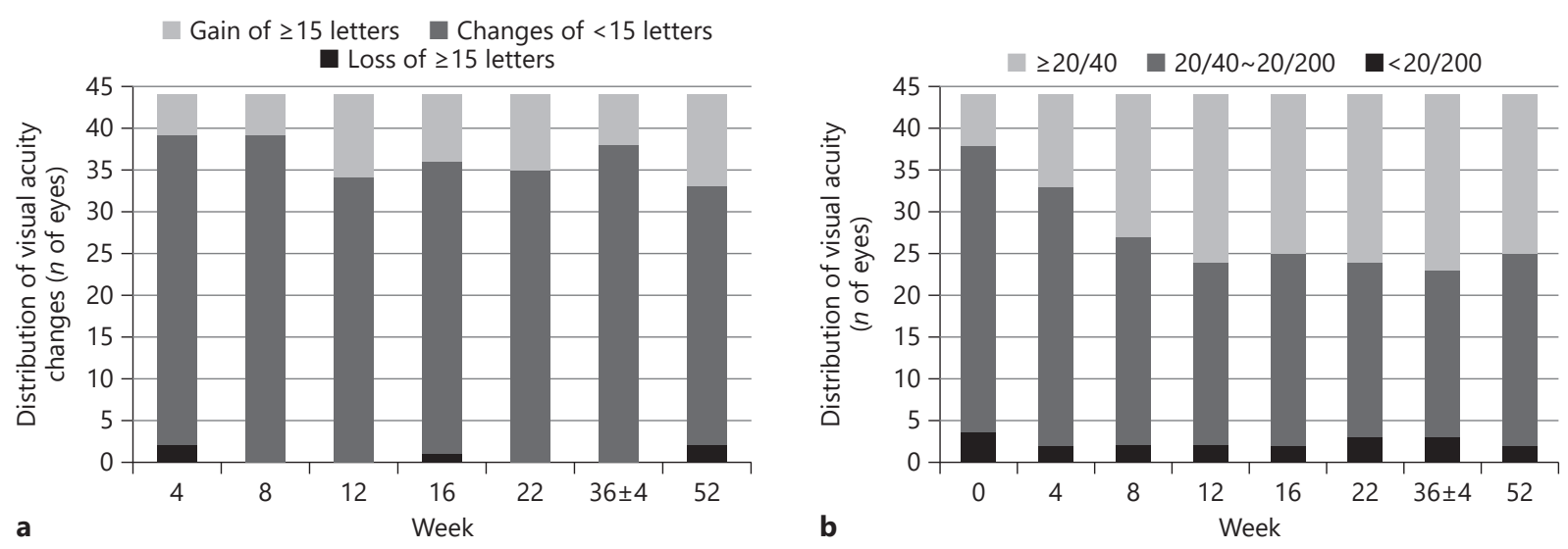

Fig. 3. Distribution of visual acuity in terms of changes in ETDRS letter score from baseline (a) and BCVA (b) for 52 weeks in diabetic macular edema treated with aflibercept using the treat-and-extend regimen.

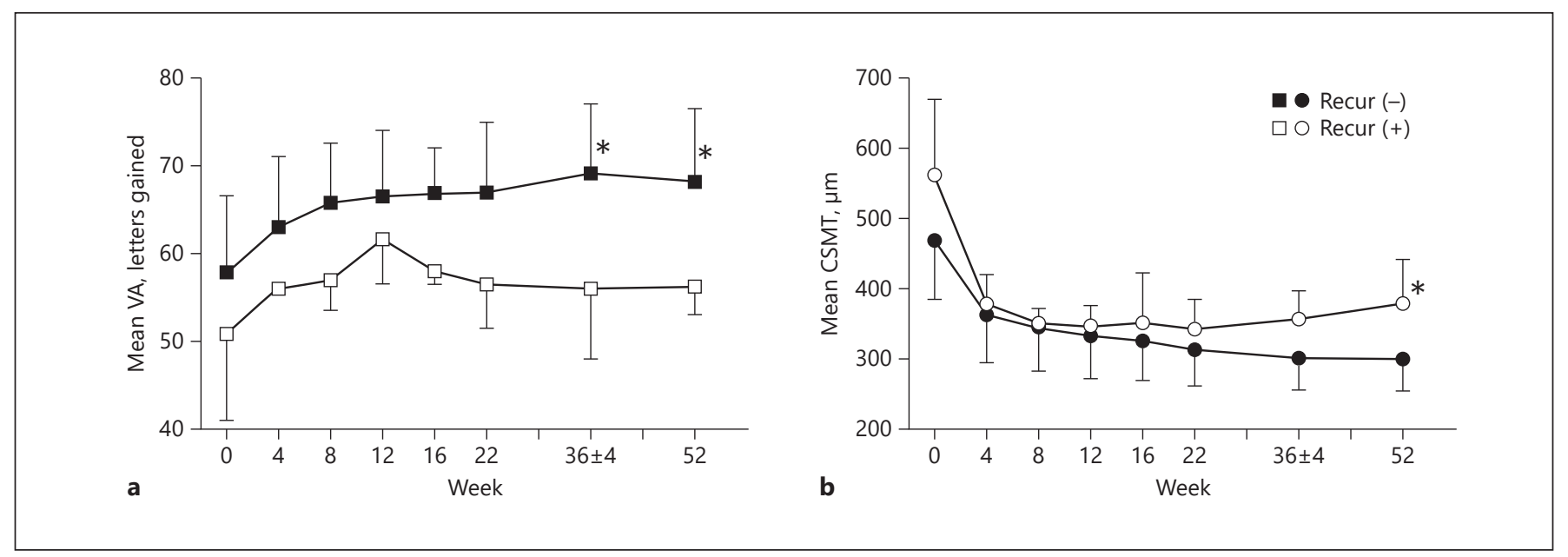

Fig. 4. Visual acuity (VA) (a) and central subfield macular thickness (CSMT) (b) according to the recurrence of diabetic macular edema during treatment with aflibercept using the treat-and-extend regimen. The asterisk indicates a significant difference between the 2 groups at the marked visit ( ${ }^{*}<0.05$, Mann-Whitney $\mathrm{U}$ test).

at 52 weeks was significantly better in the Recur (-) group $(68.1 \pm 11.5$ letters) than in the Recur (+) group (56.3 \pm 22.4 letters, $p=0.036$; Fig. 4a). Similarly, CSMT decreased in both groups significantly: $-168.5 \pm 122.6 \mu \mathrm{m}$ in the Recur $(-)$ group and $-181.7 \pm 190 \mu \mathrm{m}$ in the Recur (+) group, again with no significant differences between groups $(p=$ 0.545). However, CSMT at 52 weeks was significantly lower in the Recur $(-)$ group $(298.6 \pm 73.3 \mu \mathrm{m})$ than in the Recur (+) group (378.6 $\pm 86.4 \mu \mathrm{m}, p=0.002$; Fig. $4 \mathrm{~b})$.

The mean number of injections was lower $(8.2 \pm 0.4$ vs. $9.5 \pm 1.3$ times, $p<0.001)$ and the interval between visits

Treat-and-Extend Regimen for DME longer $(11.9 \pm 0.3$ vs. $7.3 \pm 2.4, p<0.001)$ in the Recur $(-)$ group than in the Recur (+) group at 52 weeks. There were no statistical differences in DRSS score $(p=0.268)$ and 2-step changes in DRSS level $(p=1.0)$.

\section{Discussion}

The VIBIM study investigated the efficacy of the TER in the management of DME using intravitreal aflibercept. The interim results at 52 weeks demonstrated a gain of 8.9 
Table 2. Comparison of our 1-year results with those of other studies

\begin{tabular}{|c|c|c|c|c|c|c|}
\hline & VISTA $^{\mathrm{a}} 2 \mathrm{q} 8$ & VIVID $^{\mathrm{a}} 2 \mathrm{q} 8$ & VIBIM & RETAIN $^{\mathrm{b}}$ & TREX-DMEc & Protocol $\mathrm{T}^{\mathrm{d}}$ \\
\hline $\begin{array}{l}\text { Regimen } \\
\text { Agent } \\
\text { Changes ( } n \text { of letters) }\end{array}$ & $\begin{array}{l}5 \text { loadings }+\mathrm{q} 8 \mathrm{w} \\
\text { aflibercept } \\
+10.7\end{array}$ & $\begin{array}{l}5 \text { loadings }+\mathrm{q} 8 \mathrm{w} \\
\text { aflibercept } \\
+10.7\end{array}$ & $\begin{array}{l}5 \text { loadings + TER } \\
\text { aflibercept } \\
+9.1\end{array}$ & $\begin{array}{l}3 \text { loadings + TER } \\
\text { ranibizumab } \\
+6.1\end{array}$ & $\begin{array}{l}4 \text { loadings }+ \text { TER } \\
\text { ranibizumab } \\
+9.6\end{array}$ & $\begin{array}{l}6 \text { loadings }+ \text { PRN } \\
\text { aflibercept } \\
+13.3\end{array}$ \\
\hline $\begin{array}{l}\text { A gain of } 15 \text { letters }^{\mathrm{e}} \\
\text { DRSS ( } \geq 2 \text {-step improvement })^{\mathrm{e}}\end{array}$ & $\begin{array}{l}31.1 \\
29.1\end{array}$ & $\begin{array}{l}33.3 \\
27.7\end{array}$ & $\begin{array}{l}28.3 \\
27.5\end{array}$ & $\begin{array}{l}\text { n.a. } \\
\text { n.a. }\end{array}$ & $\begin{array}{l}\text { n.a. } \\
\text { n.a. }\end{array}$ & $\begin{array}{l}42 \\
31.2^{\mathrm{f}}\end{array}$ \\
\hline $\begin{array}{l}\text { CSMT reduction, } \mu \mathrm{m} \\
\text { Number of injections }\end{array}$ & $\begin{array}{l}185.9 \\
8.4\end{array}$ & $\begin{array}{l}183.1 \\
8.7\end{array}$ & $\begin{array}{l}171.7 \\
8.5\end{array}$ & $\begin{array}{l}110.2 \\
7 \text { (median) }\end{array}$ & $\begin{array}{l}146 \\
10.7\end{array}$ & $\begin{array}{l}169 \\
9.2\end{array}$ \\
\hline
\end{tabular}

TER, treat-and-extend; PRN, pro re nata; q8w; a fixed dosing regimen of every 8 weeks; CSMT, central subfield macular thickness; DRSS, Diabetic Retinopathy Severity Scale score; n.a., not available.

${ }^{\mathrm{a}}$ According to [8]; ${ }^{\mathrm{b}}$ according to [16]; ${ }^{\mathrm{c}}$ according to [17]; ${ }^{\mathrm{d}}$ according to [26]; ${ }^{\mathrm{e}}$ values are percentages of patients; ${ }^{\mathrm{f}}$ according to specified criteria in [27].

letters with a mean number of 8.5 injections. About a quarter of the patients gained $\geq 15$ ETDRS letters. About three-quarters of the eyes did not experience worsening of macular edema and had a 12-week interval between treatments at 52 weeks.

The VIVID and VISTA studies were the randomized clinical trials that proved the efficacy of the fixed dosing regimen using aflibercept for the treatment of DME. Fixed dosing of intravitreal aflibercept every 4 or 8 weeks provided significant improvements in visual and anatomic outcomes compared to laser photocoagulation with no differences between the $2 \mathrm{q} 4$ and $2 \mathrm{q} 8$ regimens, and the improvements observed at 52 weeks were maintained at 100 weeks and 148 weeks $[8,9,18]$. However, it is difficult to maintain the fixed dosing regimen in clinical practice, as repeating injections every 1 or 2 months can become a burden for patients, doctors, and society. In addition, most patients with DME treated with anti-VEGF maintained VA for 5 years with minimal administration of treatment after 3 years [19]. As DME seems to be stabilized with adequate treatment [20], there is a potential risk of overtreatment with the fixed dosing regimen. Accordingly, it is necessary to develop individualized dosing regimens to reduce the number of injections and visits required without losing the expected improvement in vision observed in the randomized clinical trials.

As mentioned in the introduction, the intervals between visits are adjusted while keeping the injection in a TER. Preventing overtreatment with reducing recurrence is the mainstay of the TER. A couple of studies reported the efficacy of the TER using ranibizumab in the treatment of DME. The TREX-DME study [21], which compared monthly dosing and TER, with/without laser for 2 years, reported fewer injections $(18.9,17.5$, vs. 24.7) and comparable visual and anatomic gains in the TER. The RETAIN study [16] compared TER and PRN regimens for 2 years and reported a $46 \%$ reduction in the number of clinical visits with no significant differences in treatment burden or visual outcome. As aflibercept has a stronger affinity than ranibizumab for VEGF-A, as well as an affinity for VEGF-B and PIGF [7, 22], it is expected that treatment with aflibercept will require fewer injections and involve extended treatment intervals compared with the previous studies that used ranibizumab. Stratification in the Protocol T study revealed that aflibercept had superior efficacy in a subgroup with a baseline VA $\leq 20 / 50$ [23]. However, no significant differences were found in TER for exudative ARMD between ranibizumab and aflibercept in terms of treatment interval and functional outcomes [24]. The data failed to compare the 2 drugs in TER for DME as there was no report about TER using aflibercept in DME.

For the VIBIM study, we adopted almost the same inclusion criteria as the pivotal VIVID and VISTA studies, and our findings at 1 year are comparable with both (Table 2) [8]. Visual results at 52 weeks demonstrated a gain of 8.9 letters, comparable to VIVID (+10.7 of 2 q8) and VISTA ( +10.7 of $2 \mathrm{q} 8)$ studies of the fixed dosing regimen. The proportion of eyes that gained $\geq 10$ or $\geq 15$ letters was also comparable. Maintenance of visual outcome will be evaluated during the TnE phase of the second year. Improvement in CSMT was maintained throughout 52 weeks. The proportion of eyes in our study that showed $\geq 2$-step improvement was $27.5 \%$, similar to the $2 \mathrm{q} 8$ regimen of VIVID and VISTA (27.7-29.1\%).

The number of injections (8.5) was similar to the fixed 2q8 dosing of VIVID (8.7) and VISTA (8.4), as the first year of these studies included a loading phase of 5 injec- 
tions. In addition, when entering the TnE phase, the next injection was performed after 6 weeks and the required number of injections may increase compared to the $2 \mathrm{q} 8$ regimen. However, a reduction in the number of injections is expected in the second year, considering that the interval between injections was extended to 12 weeks in $73.9 \%$ of eyes and the mean interval was 10.7 weeks at 52 weeks.

The Diabetic Retinopathy Clinical Research Network (DCRC.net) Protocol T adopted the PRN regimen [25]. In the aflibercept group, VA increased by 13.3 letters at 1 year. Six loading injections were performed every 4 weeks in most of the cases, and the average number of injections was 9.2. The proportions of patients that gained 15 letters (42\%) and experienced diabetic retinopathy improvement $(31 \%)$ are comparable to our study results $[25,26]$.

In studies of TER using ranibizumab, VA improved mainly during loading injections, and was maintained for 2 years $[16,21]$. Our study also demonstrated a similar pattern in the first year. The detailed protocols for the TER study using ranibizumab differed from ours with regard to loading injection numbers, treatment interval adjustment, and capped interval. A recent meta-analysis about TER described the possibility of overtreatment with this dosing approach over PRN [13], so in our study the injection was deferred if the CSMT was stable for 2 consecutive visits extended to a maximum of 12 weeks. Although our protocol seems to be more flexible for avoiding overtreatment, the risk of undertreatment also exists. The impact of the differences in the protocols will be evaluated in the 2-year outcomes.

The subgroup analysis regarding recurrence of DME during the TnE phase revealed significantly worse VA and macular thickening at 52 weeks in the group with recurrence. Although no baseline characteristics to predict recurrence were identified, the presence of the subgroup supports the premise that flexible dosing is required according to the response to treatment of the DME.

Our study had several limitations. It was a single-arm study without a control group. The number of cases was relatively small compared with pivotal studies. However, this is the first prospective study to report the treatment of DME with aflibercept using the TER.

In conclusion, the 12-month interim results of the TER in the VIBIM study show similar efficacy to those with the fixed dosing regimen, and flexible dosing does prevent overtreatment. The study is in its second year and ongoing.

\section{Statement of Ethics}

All procedures performed in the study were in accordance with the ethical standards of the Institutional Review Board/Ethics Committee at each participating hospital and with the $1964 \mathrm{Hel}-$ sinki declaration and its later amendments. Informed consent was obtained from all individual participants included in the study.

\section{Disclosure Statement}

Hyun Woong Kim: honorarium and travel grant from Bayer, Novartis. Ji Eun Lee: consultant from Allergan, Bayer, Novartis, Samsung Bioepis, honorarium from Allergan, Bayer, Novartis, Bausch \& Lomb, AbbVie, research grant from Bayer, Novartis. Min Sagong: honorarium from Allergan, Bayer, Novartis, Bausch \& Lomb, research grant from Bayer. The following authors have no financial disclosures: Kang Yeun Pak, Jae Pil Shin, Yu Cheol Kim, Sang Joon Lee, In Young Chung, Sung Who Park.

\section{Funding Sources}

Bayer Korea (Seoul, Republic of Korea) provided research fund and drugs for this study.

\section{Author Contributions}

Kang Yeun Pak and Ji Eun Lee Pak made substantial contributions to the conception or design of the work, or to the acquisition, analysis, or interpretation of data for the work, and participated in drafting the work or revising it critically for important intellectual content. All other authors made substantial contributions to the conception or design of the work, or to the acquisition, analysis, or interpretation of data for the work.

\section{References}

Treat-and-Extend Regimen for DME
1 Klein R, Klein BE, Moss SE, Cruickshanks KJ. The Wisconsin Epidemiologic Study of Diabetic Retinopathy. XV. The long-term incidence of macular edema. Ophthalmology. 1995 Jan;102(1):7-16.

2 Early Treatment Diabetic Retinopathy Study Research Group. Treatment techniques and clinical guidelines for photocoagulation of diabetic macular edema. Early
Treatment Diabetic Retinopathy Study Report Number 2. Ophthalmology. 1987 Jul;94 (7):761-74.

3 Diabetic Retinopathy Clinical Research Network. A randomized trial comparing intravitreal triamcinolone acetonide and focal/grid photocoagulation for diabetic macular edema. Ophthalmology. 2008 Sep;115(9):1447- 
4 Chew E, Strauber S, Beck R, Aiello LP, Antoszyk A, Bressler N, et al.; Diabetic Retinopathy Clinical Research Network. Randomized trial of peribulbar triamcinolone acetonide with and without focal photocoagulation for mild diabetic macular edema: a pilot study. Ophthalmology. 2007 Jun;114(6):1190-6.

5 Nguyen QD, Brown DM, Marcus DM, Boyer DS, Patel S, Feiner L, et al.; RISE and RIDE Research Group. Ranibizumab for diabetic macular edema: results from 2 phase III randomized trials: RISE and RIDE. Ophthalmology. 2012 Apr;119(4):789-801.

6 Ross EL, Hutton DW, Stein JD, Bressler NM, Jampol LM, Glassman AR; Diabetic Retinopathy Clinical Research Network. Cost-effectiveness of aflibercept, bevacizumab, and ranibizumab for diabetic macular edema treatment: analysis from the diabetic retinopathy clinical research network comparative effectiveness trial. JAMA Ophthalmol. 2016 Aug; 134(8):888-96

7 Papadopoulos N, Martin J, Ruan Q, Rafique A, Rosconi MP, Shi E, et al. Binding and neutralization of vascular endothelial growth factor (VEGF) and related ligands by VEGF Trap, ranibizumab and bevacizumab. Angiogenesis. 2012 Jun;15(2):171-85.

8 Korobelnik JF, Do DV, Schmidt-Erfurth U, Boyer DS, Holz FG, Heier JS, et al. Intravitreal aflibercept for diabetic macular edema. Ophthalmology. 2014 Nov;121(11):2247-54.

9 Brown DM, Schmidt-Erfurth U, Do DV, Holz FG, Boyer DS, Midena E, et al. Intravitreal aflibercept for diabetic macular edema: 100 week results from the VISTA and VIVID studies. Ophthalmology. 2015 Oct;122(10): 2044-52.

10 Fung AE, Lalwani GA, Rosenfeld PJ, Dubovy SR, Michels S, Feuer WJ, et al. An optical coherence tomography-guided, variable dosing regimen with intravitreal ranibizumab (Lucentis) for neovascular age-related macular degeneration. Am J Ophthalmol. 2007 Apr; 143(4):566-83.

11 Spaide R. Ranibizumab according to need: a treatment for age-related macular degeneration. Am J Ophthalmol. 2007 Apr;143(4): 679-80.

12 Stone T, Mittra RJ. American Society of Retina Specialists 2015 preferences and trends membership survey. Chicago (IL): ASRS; 2015.

13 Freund KB, Korobelnik JF, Devenyi R, Framme C, Galic J, Herbert E, et al. Treatand-extend regimens with anti-VEGF agents in retinal diseases: a literature review and consensus recommendations. Retina. 2015 Aug; 35(8):1489-506.

14 Silva R, Berta A, Larsen M, Macfadden W, Feller C, Monés J; TREND Study Group. Treat-and-extend versus monthly regimen in neovascular age-related macular degeneration: results with ranibizumab from the TREND study. Ophthalmology. 2018 Jan; 125(1):57-65.

15 Wykoff CC, Croft DE, Brown DM, Wang R, Payne JF, Clark L, et al.; TREX-AMD Study Group. Prospective trial of treat-and-extend versus monthly dosing for neovascular agerelated macular degeneration: TREX-AMD 1-year results. Ophthalmology. 2015 Dec; 122(12):2514-22.

16 Prünte C, Fajnkuchen F, Mahmood S, Ricci F, Hatz K, Studnička J, et al.; RETAIN Study Group. Ranibizumab $0.5 \mathrm{mg}$ treat-and-extend regimen for diabetic macular oedema: the RETAIN study. Br J Ophthalmol. 2016 Jun;100(6):787-95.

17 Payne JF, Wykoff CC, Clark WL, Bruce BB, Boyer DS, Brown DM; TREX-DME Study Group. Randomized trial of treat and extend ranibizumab with and without navigated laser for diabetic macular edema: TREX-DME 1-year outcomes. Ophthalmology. 2017 Jan; 124(1):74-81.

18 Gupta OP, Shienbaum G, Patel AH, Fecarotta C, Kaiser RS, Regillo CD. A treat and extend regimen using ranibizumab for neovascular age-related macular degeneration clinical and economic impact. Ophthalmology. 2010 Nov; 117(11):2134-40.

19 Heier JS, Korobelnik JF, Brown DM, SchmidtErfurth U, Do DV, Midena E, et al. Intravitreal aflibercept for diabetic macular edema: 148-week results from the VISTA and VIVID studies. Ophthalmology. 2016 Nov;123(11): 2376-85.

20 Elman MJ, Ayala A, Bressler NM, Browning D, Flaxel CJ, Glassman AR, et al.; Diabetic Retinopathy Clinical Research Network. Intravitreal Ranibizumab for diabetic macular edema with prompt versus deferred laser treatment: 5-year randomized trial results. Ophthalmology. 2015 Feb;122(2):375-81.

21 Elman MJ, Bressler NM, Qin H, Beck RW, Ferris FL 3rd, Friedman SM, et al.; Diabetic Retinopathy Clinical Research Network. Expanded 2-year follow-up of ranibizumab plus prompt or deferred laser or triamcinolone plus prompt laser for diabetic macular edema. Ophthalmology. 2011 Apr;118(4):609-14.

22 Payne JF, Wykoff CC, Clark WL, Bruce BB, Boyer DS, Brown DM; TREX-DME Study Group. Randomized trial of treat \& extend ranibizumab with \& without navigated laser versus monthly dosing for DME: TREX-DME 2-year outcomes. Am J Ophthalmol. 2019; 202:91-9.

23 Holash J, Davis S, Papadopoulos N, Croll SD, Ho L, Russell M, et al. VEGF-Trap: a VEGF blocker with potent antitumor effects. Proc Natl Acad Sci USA. 2002 Aug;99(17):113938.

24 Wells JA, Glassman AR, Ayala AR, Jampol LM, Bressler NM, Bressler SB, et al; Diabetic Retinopathy Clinical Research Network. Aflibercept, bevacizumab, or ranibizumab for diabetic macular edema: two-year results from a comparative effectiveness randomized clinical trial. Ophthalmology. 2016 Jun; 123(6):1351-9.

25 Gillies MC, Hunyor AP, Arnold JJ, Guymer $\mathrm{RH}$, Wolf S, Ng P, et al. Effect of Ranibizumab and Aflibercept on Best-Corrected Visual Acuity in Treat-and-Extend for Neovascular Age-Related Macular Degeneration: A Randomized Clinical Trial. JAMA Ophthalmol. 2019 Apr;137(4):372-9.

26 Diabetic Retinopathy Clinical Research Network; Wells JA, Glassman AR, Ayala AR, Jampol LM, Aiello LP, Antoszyk AN, et al. Aflibercept, bevacizumab, or ranibizumab for diabetic macular edema. N Engl J Med. 2015 Mar;372(13):1193-203.

27 Bressler SB, Liu D, Glassman AR, Blodi BA, Castellarin AA, Jampol LM, et al. the Diabetic Retinopathy Clinical Research Network: Change in Diabetic Retinopathy through 2 Years. Secondary analysis of a randomized clinical trial comparing aflibercept, bevacizumab, and ranibizumab. JAMA Ophthalmol. 2017;135:558-68. 